\title{
Exploding electron bubbles
}

The first observations of excited electron bubbles in superfluid helium reveal some puzzling features

From Peter McClintock in the Department of Physics, Lancaster University, UK

The "particle in a box" is a standard second year quantum mechanics problem, much beloved by lecturers and mastered by successive generations of undergraduates. An electron bubble in liquid helium provides a strikingly simple example of this kind of system, and theory predicts that it should exhibit a series of excited states. But these states have never been demonstrated experimentally. Now Denis Konstantinov and Humphrey Maris of Brown University in the US have observed one of the excited states for the first time by exploding electrons bubbles with sound waves (D Konstantinov and H Maris 2003 Phys. Rev. Lett. 90 025302).

When an excess electron is placed in liquid helium, it expels about 1000 helium atoms from around itself and creates a vacuum bubble that contains nothing but its own wave function. The size of the bubbles is determined by the interplay of various energy contributions. Larger bubbles reduce the zero-point energy of the electron -the minimum energy that a particle can have due to quantum-mechanical uncertainty -- but at the same time have a greater surface energy. If there is a finite ambient pressure then the volume energy of the bubble will also increase. There is therefore an optimal bubble size that minimises the total energy. When the electron is in its $1 \mathrm{~s}$ ground state, the resultant bubble is spherical with a radius of 1.9::nm for zero applied pressure. With its negative electronic charge, the bubble provides a semimacroscopic object that is often somewhat misleadingly called a negative ion. 
Electron bubbles have proved to be exceedingly useful probes of superfluid ${ }^{4} \mathrm{He}$, which is otherwise hard to study because it is magnetically inert. They can readily be formed by injecting electrons into the liquid helium by field emission at a sharp metal tip, and their subsequent arrival at any point in the apparatus can be registered as a current. Electron bubbles have been used for several important experiments on superfluid ${ }^{4} \mathrm{He}$, such as a measurement of the Landau critical velocity at which superfluidity breaks down, and for studying the creation and decay of quantized vortices and superfluid turbulence. These experiments use ground state electron bubbles, in which the trapped electron is in its 1 state.

But electron bubbles also have excited states. Here the average radius and shape of the bubble will be different because the internal pressure and symmetry of the electron wave function will not be the same. Calculations of the form of the bubble for different applied pressures -- also made by the Brown group -- yield interesting results for the $1 s$ and $1 p$ states (see figure). With positive applied pressure, the vacuum--helium interface that "dresses" the electron is pressed tightly around it, and the shape of the wave function impresses itself strongly upon the bubble. This clearly seen for the $1 p$ state at a pressure of $5::$ bar. But as the pressure is reduced the bubble grows and loses its waist.

Until now it has been far from clear how any of these phenomena might be demonstrated. Maris and Konsantinov applied a strong acoustic field to the bubbles and measured the negative pressures at which they became unstable. They operated an ultrasonic transducer at 1.4::MHz, which is much slower than the few picoseconds that it takes for the bubble to equilibrate. The bubble therefore perceives the acoustic field as a quasistatic change in the ambient pressure. 
Calculations show that there is a critical reduced pressure at which the growing bubble becomes unstable and explodes because the surface tension is no longer capable of holding it together. The critical pressures are calculated as --1.89 and -1.63::bar for the $1 s$ and $1 p$ bubbles respectively.

The researchers first injected electrons into liquid helium from a tungsten tip and then used a $\mathrm{CO}_{2}$ laser to excite some of them into the $1 p$ state. Next they used the cyclic acoustic field to explode the bubbles to giant size and then shrink them again. If this change in pressure is sufficiently large, the bubbles are able to scatter enough light from a second He--Ne laser that they can be detected using a photomultiplier. In doing this, Konstantinov and Maris found that the $1 p$ bubbles apparently begin to break at a transducer voltage that is about $18 \%$ less than the for the $1 \mathrm{~s}$ bubbles, which is in reasonable agreement with the calculated value of $14 \%$.

These results are very satisfying. But Konstantinov and Maris suggest that there are even more important things to come. Their main results were obtained at $1.8:: \mathrm{K}$, but below $1.4:: \mathrm{K}$ they observed quite different and unexpected behaviour. At 1::bar, for example, the $\mathrm{CO}_{2}$ laser did not appear to produce any $1 p$ bubbles. Rather, it created new entities that were actually harder to explode than the ground state $1 \mathrm{~s}$ bubbles. They also live much longer -- in excess of $10:: m s$ compared with the $1 p$ bubble lifetime of $40:: \mu \mathrm{s}$. It is not known yet what these things are, but it is intriguing to note that they appear under just the same conditions where Maris predicted some counterintuitive effects in 2000.

At these lower temperatures there is less of the viscous normal fluid component to damp the motion of the bubble after a $1 s$ to $1 p$ transition. Thus as its shape changes from being like one of those in the top of the figure to being like one in the bottom, 
the inertia of the moving liquid will result in an overshoot. In other words, the waist on the bubble may eventually pinch off completely.

Maris's earlier suggestion that this would inevitably divide the electron into two parts has unsurprisingly proved controversial. Whether or not the recently discovered lowtemperature entities are associated with the idea of electron splitting is not known. But the next stage in the research is eagerly awaited

Fig 1. Electron bubbles in the $1 s$ (ground) state and $1 p$ state at three different pressures 